\title{
Activation of PI3K/Akt and ERK signaling pathways antagonized sinomenine-induced lung cancer cell apoptosis
}

\author{
LIPING ZHOU ${ }^{1}$, HONG LUAN ${ }^{1}$, QINGPENG LIU ${ }^{2}$, TINGSHU JIANG ${ }^{3}$, \\ HONGYUAN LIANG ${ }^{4}$, XIHUA DONG ${ }^{1}$ and HONG SHANG ${ }^{1}$ \\ ${ }^{1}$ Department of Laboratory Medicine, The First Hospital; ${ }^{2}$ Seven Years System 96K, China Medical University, \\ Shenyang, Liaoning 110001; Departments of ${ }^{3}$ Respiratory Medicine, and ${ }^{4}$ Radiology, \\ The Shengjing Hospital of China Medical University, Shenyang, Liaoning 110001, P.R. China
}

Received October 22, 2011; Accepted February 10, 2012

DOI: $10.3892 / \mathrm{mmr} .2012 .798$

\begin{abstract}
Sinomenine (SIN) is a bioactive component derived from a Chinese medicinal plant. Our previous studies demonstrated that SIN has cytotoxic effects on human lung cancer cells. However, the antitumor molecular mechanisms of SIN have yet to be elucidated in detail. In the present study, we further explored the effects of SIN on NCI-H460 human lung cancer cell viability and apoptosis and investigated the regulation and function of PI3K/Akt and ERK signaling pathways during SIN-induced apoptosis in various lung cancer cell lines. NCI-H460 cells were incubated with $200 \mu \mathrm{g} / \mathrm{ml} \mathrm{SIN}$ for the indicated times $(0,24,48$ or $72 \mathrm{~h})$. Cell viability was assessed by MTT assay. Akt, p-Akt, ERK1/2 and p-ERK1/2 protein levels were detected by western blotting, respectively. Two different selective inhibitors (LY294002 for the PI3K pathway; PD98059 for the MEK/ERK pathway) were used to characterize the relative roles of PI3K/Akt and ERK in SIN-induced apoptosis. Apoptosis was determined by flow cytometry. SIN inhibited the proliferation of NCI-H460 cells in a time-dependent manner, which was accompanied with significant activation of pAkt and pERK. LY294002 and PD98059 both significantly increased SIN-induced apoptosis in NCI-H460, NCI-H226 and NCI-H522 cells. Our findings suggest that the activation of the PI3K/Akt and ERK signaling pathways antagonize SIN-induced lung cancer cell apoptosis and molecules that inhibit these pathways should potentiate the effects of SIN. This study represents a significant step forward in our understanding of the signal transduction pathways associated with the apoptosis elicited by SIN.
\end{abstract}

Correspondence to: Dr Hong Shang, Department of Laboratory Medicine, The First Hospital of China Medical University, No. 155 North Nanjing Street, Heping, Shenyang, Liaoning 110001, P.R. China

E-mail: hongshang100@gmail.com

Abbreviations: SIN, sinomenine

Key words: sinomenine, apoptosis, lung cancer cells

\section{Introduction}

The alkaloid sinomenine (7,8-didehydro-4-hydroxy-3,7dimethoxy-17-methyl-9 $\alpha, 13 \alpha, 14 \alpha$-morphinan-6-one) (SIN) is abioactive component derived from the Chinese medicinal plant Sinomenium acutum Rehd. et Wils (family Menispermaceae), which has been utilized by Chinese doctors to treat inflammatory and arthritic diseases for more than one thousand years (1-3). Previous pharmacological studies have shown that SIN has a wide range of actions, including anti-inflammation (4-7), immunosuppression (8), arthritis amelioration (9) and protection against hepatitis induced by lipopolysaccharides (10). Furthermore, in studies using intramuscular injection and multiple dosing, a combination of SIN and cyclosporin A produced immunomodulatory effects in a cardiac transplant model (11). However, little is known concerning the antitumor effects of SIN, and the antitumor molecular mechanisms of SIN have yet to be elucidated in detail.

In our previous studies, we found that SIN has cytotoxic effects on human lung cancer cells by inducing apoptosis through mechanisms including the activation of caspase- 3 and -9 , loss of $\Delta \Psi \mathrm{m}$ and release of cytochrome $\mathrm{C}$ (12). However, it is unknown whether SIN also activates proliferation-associated signaling pathways. Additionally, it is unknown whether SIN-induced cell apoptosis is affected when proliferationassociated signaling pathways are inhibited.

Akt and ERK are important signaling molecules that promote survival in different types of cancer. In this study, we explored the effects of SIN on human lung cancer NCI-H460 cell viability and apoptosis and investigated the regulation and function of PI3K/Akt and ERK signaling pathways during SIN-induced apoptosis in various lung cancer cell lines (including NCI-H460, NCI-H226 and NCI-H522) to unravel the detailed molecular mechanisms underlying its anticancer effects.

\section{Materials and methods}

Materials and chemicals. RPMI-1640 cell culture media, antibiotics and trypsin were obtained from Biological Industries (Kibbutz Beit Haemek, Israel). Fetal bovine serum (FBS) was purchased from Solarbio (Beijing Solarbio Science and 
Technology Co., Ltd., China). 3-(4,5-Dimethyl thiazol-2yl)-2, 5-diphenyltetrazolium bromide (MTT) and the dimethyl sulfoxide (DMSO) kit was purchased from Sigma-Aldrich (St. Louis, MO, USA). Propidium iodide (PI) staining and Roh123 fluorescent dye kits were purchased from Nanjing KeyGene Biotech Co., Ltd. (Nanjing, China). SIN (HPLC purity $\geq 98 \%$ ) was purchased from the Xi'an Sobeo Pharmtech Co., Ltd. (Xi'an, China). A 2-mM stock solution of SIN was prepared in RPMI- 1640 growth medium and stored at $-20^{\circ} \mathrm{C}$. Horseradish peroxidase-conjugated secondary antibody was obtained from Santa Cruz Biotechnology, Inc. (Santa Cruz, CA). All other chemicals were obtained from Sinopharm Chemical Reagent Co., Ltd. (Shenyang, China).

Cell culture. All of the cell lines derived from human lung cancers were obtained from the China Center for the Type Culture Collection (Wuhan, China) and were cultured in RPMI-1640 medium supplemented with $10 \%$ fetal bovine serum, $100 \mathrm{U} / \mathrm{ml}$ penicillin, and $100 \mathrm{mg} / \mathrm{ml}$ streptomycin. Cultures were maintained in a humidified incubator at $37^{\circ} \mathrm{C}$ in the presence of $5 \% \mathrm{CO}_{2}$. Culture medium was changed every 2 days. For assays, cells were detached using a solution of $0.125 \%$ trypsin and $0.02 \%$ EDTA.

MTT assay. The effect of SIN on NCI-H460 cell proliferation in vitro was determined by measuring MTT dye absorbance of living cells. In brief, cells $\left(1 \times 10^{4}\right.$ cells/well) were seeded in 96-well tissue culture plates. Cells were cultured with RPMI-1640 growth medium for $24 \mathrm{~h}$, and then the medium was replaced with RPMI-1640 growth medium containing $200 \mu \mathrm{g} / \mathrm{ml} \mathrm{SIN}$. After exposure to SIN for $0,24,48$ or $72 \mathrm{~h}$, $25 \mu 1$ of MTT solution ( $2 \mathrm{mg} / \mathrm{ml}$ in PBS) was added to each well and the plates were incubated for an additional $4 \mathrm{~h}$ at $37^{\circ} \mathrm{C}$. The MTT solution in the medium was aspirated off, and $150 \mu 1$ of DMSO was added to each well to solubilize the formazan crystals formed in the viable cells. Optical density was measured at $570 \mathrm{~nm}$ using an ELISA plate reader (Model 550, Bio-Rad, USA). Each plate contained multiple wells for a given experimental condition and multiple control wells. This procedure was repeated three times.

Flow cytometric analysis. After treatment with $200 \mu \mathrm{g} / \mathrm{ml} \mathrm{SIN}$ for $48 \mathrm{~h}$, the human lung cancer cells were harvested and fixed with ice-cold $70 \%(\mathrm{v} / \mathrm{v})$ ethanol for $24 \mathrm{~h}$. After centrifugation at $200 \mathrm{x} \mathrm{g}$ for $5 \mathrm{~min}$, the cell pellet was washed with PBS $(\mathrm{pH}$ 7.4) and resuspended in PBS containing PI $(10 \mu \mathrm{g} / \mathrm{ml})$ and DNase-free RNase $(20 \mu \mathrm{g} / \mathrm{ml})$. Cells were then incubated at room temperature in the dark for $30 \mathrm{~min}$, and DNA content was determined by flow cytometry using a FACScan flow cytometer (Becton-Dickinson, San Jose, CA, USA). The data were analyzed using MODIFIT software (Becton-Dickinson) (20). Control cells were treated with medium only.

Western blot analysis of apoptosis-related proteins. Expression of apoptosis-related proteins was evaluated by western blotting. In brief, $3 \times 10^{7}$ cells were incubated with $200 \mu \mathrm{g} / \mathrm{ml}$ SIN for $48 \mathrm{~h}$. The cells were then washed in PBS and were suspended in five volumes of lysis buffer (20 mM HEPES, pH 7.9, 20\% glycerol, $200 \mathrm{mM} \mathrm{KCl,} 0.5$ mM EDTA, $0.5 \%$ NP40, $0.5 \mathrm{mM}$ DTT, $1 \%$ protease inhibitor cocktail). Lysates

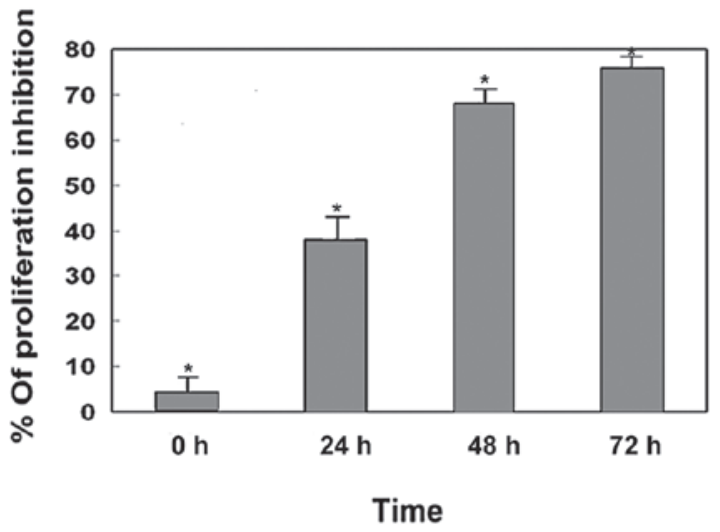

Figure 1. Effect of SIN on the proliferation of NCI-H460 cells. Cells were incubated with $200 \mu \mathrm{g} / \mathrm{ml}$ SIN for the indicated times $(0,24,48$ or $72 \mathrm{~h}$ ). The figure shows the percentage of proliferation inhibition in a time-dependent cytotoxicity assay. The results are from at least three independent experiments.

A

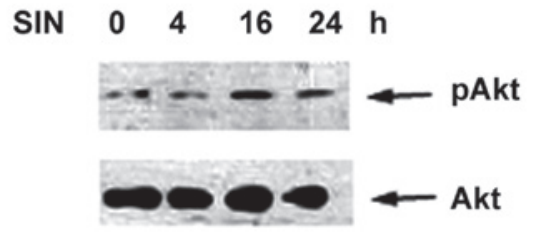

B

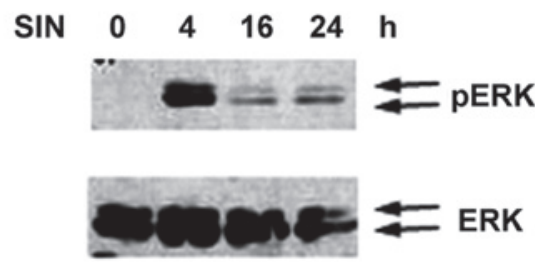

Figure 2. Activation of Akt and ERK in SIN-induced apoptosis demonstrating the levels of pAkt and pERK protein. NCI-H460 cells were treated with SIN for $0,4,16$ or $24 \mathrm{~h}$, and cell lysates were subsequently separated using SDS-PAGE and blotted with (A) anti-pAkt or anti-Akt, (B) anti-pERK1/2 or anti-ERK1/2 antibodies. The results are from at least three independent experiments.

were then collected and stored at $-20^{\circ} \mathrm{C}$ until further use. The supernatant protein concentration was determined by the Bradford method. Supernatant samples containing $40 \mu \mathrm{g}$ of total protein were resolved by SDS-PAGE based on the target protein sizes, transferred to polyvinylidene fluoride membranes by electroblotting, and probed with anti-pAkt, anti-Akt, anti-pERK and anti-ERK. Membranes were incubated with horseradish peroxidase-conjugated secondary antibodies. Blots were developed using an ECL kit.

Statistical analysis. All experiments were conducted three times. The results were expressed as mean and standard deviation and evaluated using one- or two-way ANOVA followed by the Student's t-test. The statistical significance was defined as $\mathrm{P}<0.05$. 


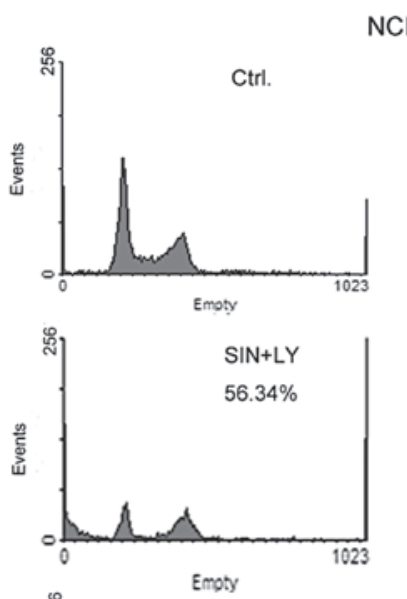

$\mathrm{NCl}-\mathrm{H} 460$
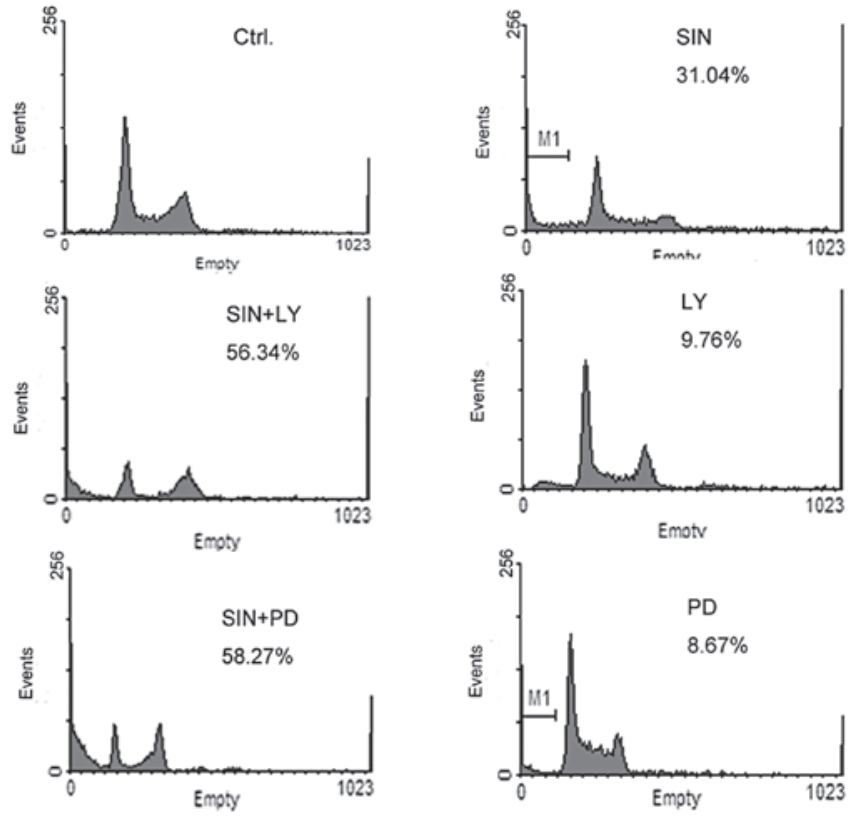

Figure 3. Effects of a PI3K/Akt inhibitor (LY) and a MEK/ERK inhibitor (PD) on SIN-treated NCI-H460 cells. NCI-H460 cells were treated with the indicated reagents for $48 \mathrm{~h}$ and stained with PI. Apoptosis was then analyzed by flow cytometry. The final results are summarized in the column graph, and the results are from at least three independent experiments.

\section{Results}

Effects of SIN on NCI-H460 cell viability and apoptosis. To investigate the potential cell proliferation inhibitory activity of SIN, NCI-H460 cells were treated with $200 \mu \mathrm{g} / \mathrm{ml} \mathrm{SIN} \mathrm{for}$ the indicated times. As confirmed by MTT assay, SIN trig- gered time-dependent cytotoxicity, resulting in a significant loss of cell viability (Fig. 1). These results indicate that the influence of SIN on the inhibition of NCI-H460 cell proliferation in vitro is mainly due to time-dependent apoptotic cell death.

Activation of Akt and ERK in SIN-induced apoptosis. The activation of the Akt and ERK1/2 pathways is normally associated with cell proliferation and survival (13-15). However, the role of Akt and ERK1/2 activation in SIN-induced apoptosis remains somewhat unclear. Therefore, to determine whether PI3-K/Akt and ERK pathways were involved in the regulation of SIN chemosensitivity, NCI-H460 cells were treated with SIN, and western blotting was performed. Akt phosphorylation was maximally increased at $16 \mathrm{~h}$, whereas protein levels of total Akt were not altered (Fig. 2A). Furthermore, ERK was also significantly activated, and this activation peaked at $4 \mathrm{~h}$ (Fig. 2B). These results indicate that both Akt and ERK activation may be involved in SIN-induced apoptosis.

Effects of a PI3K/Akt inhibitor and a MEK/ERK inhibitor on SIN-treated NCI-H460 cells. To characterize the relative roles of Akt and ERK in SIN-induced apoptosis, two different selective inhibitors (LY294002 for the PI3K pathway; PD98059 for the MEK/ERK pathway) were used. SIN treatment alone increased apoptosis when compared with the control-treated cells. Furthermore, co-treatment with SIN plus the PI3K/ Akt inhibitor LY294002 significantly increased apoptosis by $25.3 \%$ over SIN treatment alone (Fig. 3). NCI-H460 cells co-treated with SIN and the ERK inhibitor also exhibited a synergistic effect on apoptosis (Fig. 3). Therefore, our results strongly suggest that the inactivation of PI3K/Akt may play an important role in SIN-induced apoptosis. Moreover, our results suggest that the activation of Akt and ERK is functionally
A
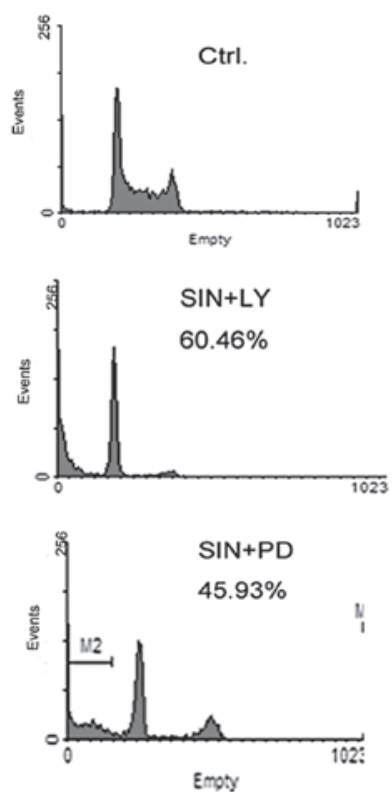

$\mathrm{NCl}-\mathrm{H} 226$
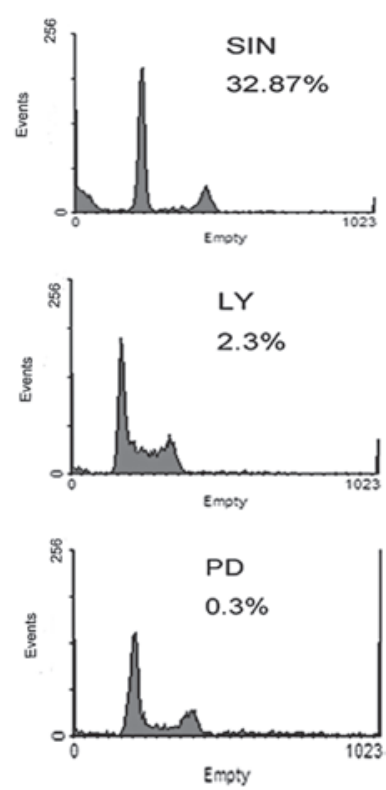

B
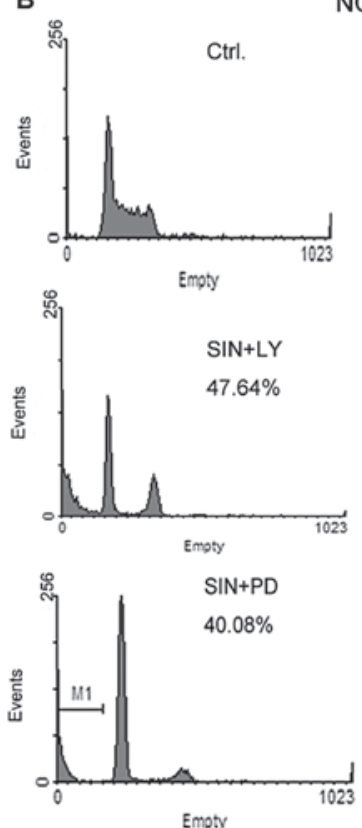

$\mathrm{NCl}-\mathrm{H} 522$
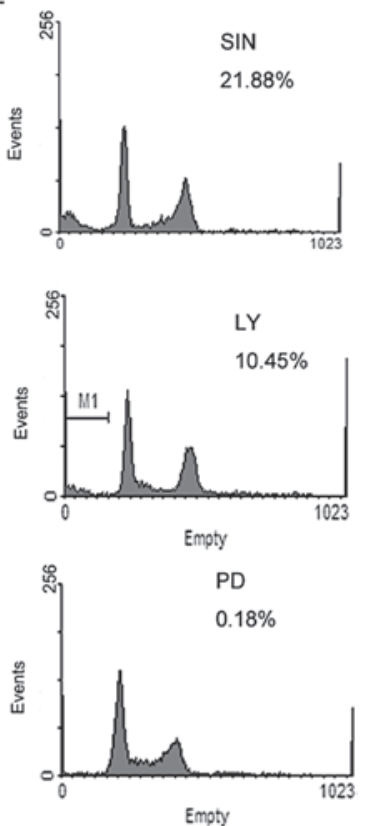

Figure 4. Effects of LY294002 and PD98059 on two addition SIN-treated lung cancer cell lines. (A) NCI-H226 and (B) NCI-H522 were incubated with SIN alone, SIN plus LY294002 (LY), SIN plus PD98059 (PD), LY294002 alone or PD98059 alone. FACScan flow cytometry was then used to detect the proportion of apoptotic cells. The results are from at least three independent experiments. 
involved in the negative regulation of SIN-induced apoptosis in NCI-H460 cells.

Effects of LY294002 and PD98059 on two additional SIN-treated lung cancer cell lines. To further confirm that the Akt and ERK signaling pathways play a critical role in the process of SIN-induced cell apoptosis, we employed two additional lung cancer cell lines, NCI-H226 and NCI-H522. These cell lines were incubated with SIN alone, SIN plus LY294002, SIN plus PD98059, LY294002 alone, or PD98059 alone. Using FACScan analysis, we quantitatively assessed apoptosis in these cancer cells under the various treatment conditions. Fig. 4 shows that SIN markedly inhibited growth of NCI-H226 and NCI-H522 cells. We also found that the two-drug combination treatment resulted in more apoptotic cells than either single-drug treatment alone. Specifically, when combined with LY294002, SIN-induced apoptosis increased from 32.87 to $60.46 \%$ in the NCI-H226 cell line and from 21.88 to $47.64 \%$ in the NCI-H522 cell line $(\mathrm{P}<0.05)$ (Fig. 4). Cells co-treated with SIN and PD98059 also exhibited such synergistic effects $(\mathrm{P}<0.05)$ (Fig. 4). These results indicate that both Akt and ERK pathways are essential for SIN-induced cell apoptosis in multiple lung cancer cell lines.

\section{Discussion}

Lung cancer is the most prevalent form of cancer worldwide. Five-year survival is achieved by approximately $10-15 \%$ of patients (16). Therefore, the search for new chemopreventive and antitumor agents is still an urgent clinical problem.

SIN is a pure alkaloid extracted from a Chinese medicinal plant. Although it has been used clinically as an anti-inflammatory agent in several inflammation-related diseases $(5,6,17)$, to date few studies have addressed the anti-cancer mechanism of this herbal medicine. A recent study showed that SIN exhibits a significant apoptotic effect on hematoma carcinoma cells (18). In addition, SIN was shown to significantly inhibit proliferation of IL-1 $\beta$-activated human synovial sarcoma cells (Hs701.T) (19). In our previous study, we found that SIN exerts a significant apoptotic effect on NCI-H460 cells through several apoptosis-associated signaling mechanisms, such as the collapse of the mitochondrial membrane potential, the release of cytochrome $C$ and the activation of caspase- 9 and -3 (12). In the present study, we further confirmed that SIN inhibits the proliferation of NCI-H460 cells and induces cell apoptosis in a time-dependent manner. Moreover, SIN-induced cell apoptosis was observed not only in the NCI-H460 cell line but also in the NCI-H226 and NCI-H522 cell lines.

As Akt is a crucial mediator of carcinogenesis and the phosphorylation of Akt is essential for its full activity (20-22), we measured the potential effects of SIN on Akt phosphorylation. As shown in Fig. 2A, Akt phosphorylation occurred in SIN-induced apoptosis, while suppression of Akt activation by LY294002 significantly upregulated cell apoptosis (Figs. 3 and 4). ERK is also known to be a critical factor in carcinogenesis and is closely associated with Akt signaling (23-26); therefore, we measured the potential effects of SIN treatment on ERK signaling. Similar to Akt, the inhibition of ERK activation by PD98059 resulted in a significant synergistic effect on apoptosis such that the degree of apoptosis was much higher than with SIN treatment alone (Fig. 3). These results suggest that suppression of PI3K/Akt and ERK activity is responsible for the significantly elevated levels of SIN-induced apoptosis. Considering this, we evaluated the proliferation-associated signaling pathways related to SIN-induced apoptosis.

In conclusion, taken together, our findings indicate that the activation of PI3K/Akt and ERK signaling pathways antagonize SIN-induced lung cancer cell apoptosis, and molecules that inhibit these pathways should potentiate the effects of SIN. This study represents a significant step forward in our understanding of the signal transduction pathways associated with the apoptosis elicited by SIN. However, further in vivo studies are warranted to verify the therapeutic efficacy of SIN in the treatment of lung cancer.

\section{Acknowledgements}

This study was supported by a grant from the Science and Technology Research Project of the Higher Education Department of Liaoning Province (no. L2010695).

\section{References}

1. Wong LC and Wu LT: History of Chinese Medicine. National Quarantine Service, Shanghai, 1936 (In Chinese).

2. Yamasaki H: Pharmacology of sinomenine, an anti-rheumatic alkaloid from Sinomenium acutum. Acta Med Okayama 30: 1-20, 1976.

3. Kong QF and Li J: New study progress of sinomenine. Zhongguo Zhong Yao Za Zhi 30: 1573-1576, 2005 (In Chinese).

4. Wang Y, Zhou L and Li R: Study progress in Sinomenium acutum (Thunb.) Rehd. et Wils. Zhong Yao Cai 25: 209-211, 2002 (In Chinese)

5. Kok T, Yue P, Mak N, Fan T, Liu L and Wong R: The antiangiogenic effect of sinomenine. Angiogenesis 8: 3-12, 2005.

6. Qian L, Xu Z, Zhang W, Wilson B, Hong JS and Flood PM: Sinomenine, a natural dextrorotatory morphinan analog, is anti-inflammatory and neuroprotective through inhibition of microglial NADPH oxidase. J Neuroinflammation 4: 23-37, 2007.

7. Xu M, Liu L, Qi C, Deng B and Cai X: Sinomenine versus NSAIDs for the treatment of rheumatoid arthritis: a systematic review and meta-analysis. Planta Med 74: 1423-1429, 2008.

8. Vieregge B, Resch K and Kaever V: Synergistic effects of the alkaloid sinomenine in combination with the immunosuppressive drugs tacrolimus and mycophenolic acid. Planta Med 65: 80-82, 1999.

9. Liu L, Resch K and Kaever V: Inhibition of lymphocyte proliferation by the anti-arthritic drug sinomenine. Int J Immunopharmacol 16: 685-691, 1994.

10. Kondo Y, Takano F, Yoshida K and Hojo H: Protection by sinomenine against endotoxin-induced fulminant hepatitis ingalactosamine-sensitized mice. Biochem Pharmacol 48: 1050-1052, 1994.

11. Candinas D, Mark W, Kaever V, Miyatake T, Koyamada N, Hechenleitner P and Hancock WW: Immunomodulatory effects of the alkaloid sinomenine in the high responder ACI-to-Lewis cardiac allograft model. Transplantation 62: 1855-1860, 1996.

12. Jiang TS, Zhou LP, Zhang WL, Qu D, Xu XM, Yang Y and Li S: Effects of sinomenine on proliferation and apoptosis in human lung cancer cell line NCI-H460 in vitro. Mol Med Reports 3: 51-56, 2010.

13. Lee ER, Kim JY, Kang YJ, Ahn JY, Kim JH, Kim BW, Choi HY, Jeong MY and Cho SG: Interplay between PI3K/Akt and MAPK signaling pathways in DNA-damaging drug-induced apoptosis. Biochim Biophys Acta 1763: 958-968, 2006.

14. Cho HS, Chang SH, Chung YS, Shin JY, Park SJ, Lee ES, et al: Synergistic effect of ERK inhibition on tetrandrine-induced apoptosis in A549 human lung carcinoma cells. J Vet Sci 10: 23-28, 2009. 
15. Meng J, Dai B, Fang B, Bekele BN, Bornmann WG, Sun D, Peng Z, Herbst RS, Papadimitrakopoulou V, Minna JD, et al: Combination treatment with MEK and AKT inhibitors is more effective than each drug alone in human non-small cell lung cancer in vitro and in vivo. PLoS One 5: e14124, 2010.

16. Szczuka I and Roszkowski-Śliż K: Lung cancer in Poland in 1970-2004. Pneumonol Alergol Pol 76: 19-28, 2008 (In Polish).

17. Yan LC, Bi EG, Lou YT, Wu XD, Liu ZD, Zhou J, Wang Y, Ma Z, Lin GM, Sun SH, et al: Novel sinomenine derivative 1032 improves immune suppression in experimental autoimmune encephalomyelitis. Biochem Biophys Res Commun 391: 1093-1098, 2010.

18. Gong L and Gao Y: Apoptotic derivation of hepatoma carcinoma cell by kukoline. J Chengdu Univ Tradit Chin Med 30: 38-39, 2007 (In Chinese)

19. Li XJ, Yue PY, Ha WY, Wong DY, Tin MM, Wang PX, Wong RN and Liu L: Effect of sinomenine on gene expression of the IL-1 beta-activated human synovial sarcoma. Life Sci 79: 665-673, 2006.

20. Hövelmann S, Beckers TL and Schmidt M: Molecular alterations in apoptotic pathways after PKB/Akt-mediated chemoresistance in NCI H460 cells. Br J Cancer 90: 2370-2377, 2004.

21. Fry MJ: Phosphoinositide 3-kinase signalling in breast cancer: how big a role might it play? Breast Cancer Res 3: 304-312, 2001
22. Izycka-Swieszewska E, Brzeskwiniewicz M, Wozniak A, Drozynska E, Grajkowska W, Perek D, Balcerska A, Klepacka T and Limon J: EGFR, PIK3CA and PTEN gene status and their protein product expression in neuroblastic tumours. Folia Neuropathol 48: 238-245, 2010.

23. Lin X, Böhle AS, Dohrmann P, Leuschner I, Schulz A, Kremer B and Fändrich F: Overexpression of phosphatidylinositol 3-kinase in human lung cancer. Langenbecks Arch Surg 386: 293-301, 2001

24. Perkinton MS, Ip JK, Wood GL, Crossthwaite AJ and Williams RJ: Phosphatidylinositol 3-kinase is a central mediator of NMDA receptor signalling to MAP kinase (Erk1/2), Akt/PKB and CREB in striatal neurones. J Neurochem 80: 239-254, 2002.

25. He X, Wang J, Guo Z, Liu Q, Chen T, Wang X and Cao X: Requirement for ERK activation in sinomenine-induced apoptosis of macrophages. Immunol Lett 98: 91-96, 2005.

26. Lu ZJ, Zhou Y, Song Q, Qin Z, Zhang H, Zhou YJ, Gou LT, Yang JL and Luo F: Periplocin inhibits growth of lung cancer in vitro and in vivo by blocking AKT/ERK signaling pathways. Cell Physiol Biochem 26: 609-618, 2010. 